\title{
Proposal of a Mobile Learning Preferences Model
}

\author{
doi:10.3991/ijim.v4i4.1445 \\ J. Y-K. Yau and M. S. Joy \\ University of Warwick, Coventry, U.K.
}

\begin{abstract}
A model consisting of five dimensions of mobile learning preferences - location, level of distractions, time of day, level of motivation and available time - is proposed in this paper. The aim of the model is to potentially increase the learning effectiveness of individuals or groups by appropriately matching and allocating mobile learning materials/applications according to each learner's type. Examples are given. Our current research investigations relating to this model are described.
\end{abstract}

Index Terms-mobile learning, m-learning preferences model

\section{INTRODUCTION}

A learning style is defined as a "description of the attitudes and behaviours that determine our preferred way of learning” [1]. It has been repeatedly documented by many researchers that, when learners are taught with specific approaches matching their learning styles/preferences as identified by the Dunn and Dunn model [2], they "demonstrate statically higher achievements and aptitude test scores ... than when they are taught with approaches that mismatch their preferences" [3]. It has been argued that the personalisation of materials according to learning styles in web-based learning environments has potential to improve the learner's learning effectiveness. Using learning styles to personalise web-based learning is more prevalent than using them to personalise mobile learning (hereafter, abbreviated as m-learning) applications [4,5,8]. "When interacting with the web, not all users have the same goals, interests or needs. It is well-known that aspects such as background, goal, preferences, learning styles, or personality can influence the way in which they interact with information" [6]. In this paper, we propose that the use of learning preferences in m-learning applications is equally important, if not more so, due to the nonstationary nature of m-learning. In particular, we propose a model of mobile learning preferences (MLPs), which accommodates some of the different MLPs that mobile learners may have. The construction of the model is based on our previous work, which includes an interview study [7] and the construction of a personalized m-learning application, which deploys three of the five different dynamic MLP dimensions - location, perceived level of distractions, and time of day [8]. No existing models of MLPs have been identified at the time of writing.

This paper is structured as follows. In section 2, a literature review is presented, and in section 3, our proposed model of MLPs is illustrated. In section 4, our current research investigations are described. Finally in section 5, conclusions are given.

\section{LITERATURE REVIEW}

Specific research on MLPs, and suggesting/adapting learning materials to students based on these, have currently not been identified. There is, however, previous research relating to learning styles/preferences deployed in web-based learning environments, and the use of learning contexts in m-learning environments.

First, research has been conducted on the use of learning styles/preferences in a web-based learning environment, particularly in the areas of adaptivity and personalization. These refer to the "function enabling the system to fit the students' current situation, needs, and characteristics, taking into account, for example, their knowledge level, learning styles, cognitive abilities, current location, motivation, interests, preferred language, and so on" [9]. Adaptivity refers to the automatic customization of learners' materials by the system whereas personalization is a more generic term that includes systematic adaptations as well as issues which can be specified by learners themselves and are adapted by the system accordingly, such as user interface.

Second, research and applications have been completed and developed respectively based on the use of m-learning contexts such as those described in Yau and Joy [7]. Some of these applications have focused on suggesting appropriate learning materials (such as languages, science, animals, museum artifacts) for learners based on their situation as defined by the learning contexts stipulated by the application designers/developers. Graf and Kinshuk [9] have also described providing adaptivity and personalization in ubiquitous m-learning systems using various learning contexts information.

\section{A PROPOSED MODEL OF M-LEARNING PREFERENCES}

We propose a model consisting of five different dimensions of MLPs, namely location of study, perceived level of distractions, time of day, motivation level of the learner, and available time, as shown in table 1. Participants of our interview study have described their learning patterns/styles [7] and we have found it useful to map these onto a model consisting of five MLP dimensions. Note that other preferences were commented on, however we considered these of secondary importance in an mlearning context. The benefits of this model include a) construction of personalized m-learning applications, and b) appropriate matching of m-learning applications which suit learners' m-learning requirements. Some aspects of the five dimensions were mentioned in the Dunn and Dunn model [2] and further information relating to this are presented in Yau and Joy $[7,8]$. More dimensions may be added to the model, if they are of primary importance concerning m-learning. When matching appropriate mate 
TABLE I.

A PROPOSED MODEL OF M-LEARNING PREFERENCES DIMENSIONS

\begin{tabular}{|c|c|c|}
\hline Dimension & Description & M-learning Preferences \\
\hline $\begin{array}{l}\text { Location of } \\
\text { study }\end{array}$ & $\begin{array}{l}\text { Determining factors may include availability of resources or aca- } \\
\text { demic help, motivation by working peers, relaxing and comfortable } \\
\text { elements, maximising available time, familiarity of location, allows } \\
\text { for routine, and convenience. }\end{array}$ & $\begin{array}{l}\text { a) Study-designated areas (study alone or with peers); b) } \\
\text { Study with peers (location not specified); c) Study in } \\
\text { presence of others (e.g. in cafes); d) Making use of idle } \\
\text { time (e.g. in transport); e) Indifferent }\end{array}$ \\
\hline $\begin{array}{c}\text { Perceived } \\
\text { level of } \\
\text { distractions }\end{array}$ & $\begin{array}{l}\text { Determining factors may include noise level, how busy the environ- } \\
\text { ment is, the learner's concentration level, and the level of interrup- } \\
\text { tion at the location. }\end{array}$ & $\begin{array}{l}\text { a) High; b) Medium; c) Low; d) With 'distractors' (e.g. } \\
\text { such as music or other distractions) [10]; e) Indifferent. }\end{array}$ \\
\hline Time of day & $\begin{array}{l}\text { Determining factors may include biological clock - awakeness or } \\
\text { alertness during different parts of the day. }\end{array}$ & $\begin{array}{l}\text { a) Morning; b) Afternoon; c) Evening; d) Night; e) Indif- } \\
\text { ferent }\end{array}$ \\
\hline $\begin{array}{l}\text { Learner's } \\
\text { level of } \\
\text { motivation }\end{array}$ & $\begin{array}{l}\text { Determining factors may include intrinsic and extrinsic motivations, } \\
\text { urgency of task, pressure of performing well, and how enthusiastic } \\
\text { the learner is towards learning/m-learning/mobile devices. }\end{array}$ & $\begin{array}{l}\text { a) High; b) Medium; c) Low; d) Conditional; e) Fluctu- } \\
\text { ating; f) Indifferent }\end{array}$ \\
\hline $\begin{array}{l}\text { Available } \\
\text { time }\end{array}$ & $\begin{array}{c}\text { Determining factors may include productivity level of learner and } \\
\text { tiredness. }\end{array}$ & $\begin{array}{l}\text { a) >=60mins; b) ca45mins; c) ca30mins; d) ca15mins; e) } \\
<=10 \text { mins; f) Indifferent }\end{array}$ \\
\hline
\end{tabular}

rials/activities to mobile learners, other learning styles dimensions and factors may also be considered such as knowledge level, visual/verbal styles and concentration level. More difficult learning materials can be recommended to learners with a higher motivation to learn when this is appropriate. Highly motivated learners may be recommended to use self-regulated application, whereas individual and collaborative m-learning applications can be recommended to learners who prefer to study alone and with peers respectively.

\section{CURRENT RESEARCH INVESTIGATIONS}

We are currently conducting two research investigations relating to our proposed model of MLPs. The two corresponding objectives to these investigations are as follows - a) extending and validating this model by means of empirical studies, and b) ascertaining any similarities and/or differences of MLPs between various national cultural groups, based on this model.

\section{A. Extension and validation of the model}

We are interested in the study of MLPs because different learners may desire to study in different locations, for example under different levels of distractions and during different times of the day [7]. Helping mobile learners to know about their MLPs, and suggesting appropriate materials to them based on these preferences, can potentially enhance their learning experiences and outcomes. The model can be deployed for identifying the MLPs of a learner, which in turn can then be used a) to gain understanding of the m-learning requirements of the mobile learner, and b) to assign/recommend appropriate learning materials/activities/software applications to the learner, subject to the dimensions of the model. The validation of such a model will contribute significantly to the pedagogical aspects of m-learning.

Our research question for this objective is as follows:

- Can a comprehensive model of m-learning preferences dimensions be constructed, which is representative of different m-learning individuals and learner groups?

Our expected deliverables are as follows:

- An m-learning preferences questionnaire that guides students to ascertain their MLPs.
- A comprehensive model of MLPs dimensions, which is representative of different m-learning individuals and learner groups.

- Software design of an m-learning application to accommodate the MLPs of different learners in general.

Our validation process will include designing and developing a suitable MLPs questionnaire based on the model, and subsequently obtaining a large and diverse set of students to complete it. The results will then be analyzed to a) ensure that all groups of mobile learners can be represented and described in the model, and b) explain particular patterns of m-learning behavior concerning different mobile learner individuals or groups. The model can also be extended to include 1) a mobile devices preferences dimension (including connectivity to the Internet), 2) a national cultural preferences/characteristics dimension (including the popularity of using mobile devices for learning, specific learning trends, peer/society acceptance rates of using mobile devices for learning in the private and public domain). These further additional dimensions to be added to the model can be validated in conjunction with the proposed model as a whole.

\section{B. Eliciting similarities/differences of MLPs between learners of different national cultural groups, if any}

We are interested in different national cultural groups and how students in groups learn or m-learn. For example, there may be vast differences between how a Japanese student learns, or prefers to learn, compared with a UK student. We are particularly interested in finding out these differences and similarities, if any. We propose to collect large data samples of students concerning their MLPs from different national cultural groups, for example, UK, Sweden, Germany, South Africa and Japan. Note that the countries of investigation are to be confirmed after careful consideration, in order to obtain a representative data sample as well as interesting and useful results. The rationale for this objective is so that 1) similarities between MLPs within certain national cultural groups can be illustrated, and 2) differences can be extracted and comparisons made between cross-national cultural groups. These similarities and differences can be used to help us understand and obtain reasons to 1) why individual or group uptakes of m-learning are more successful or prevalent in certain countries than others, and 2) why some individuals 
or groups are more willing and enthusiastic about the use of mobile devices for learning than others.

Results from Yau and Joy [7] suggest that there is a variety of MLPs exhibited by UK students. For example, results showed that some students were very enthusiastic about m-learning - some thought they might potentially use them when there was a need, and some did not feel the need the use mobile devices for learning whatsoever (e.g. because they did not want to study outside working hours). We give the following example to illustrate more precisely what we mean. In Asian countries such as Japan and Taiwan, there is a much larger penetration ratio of mobile technologies that are provided by schools/institutions for students to learn with. It also seems that these groups of students

- are more open and willing to use mobile technologies for learning;

- learn/study more irrespective of time and location and outside school hours as part of their everyday life; and

- want to engage with learning/studying as often and as much as possible.

Many of the publications in the past and present proceedings of the IEEE International Conference on Wireless, Mobile, and Ubiquitous Technologies (WMUTE) confirm these observations $[11,12,13,14,15]$.

Our research question for this objective is as follows:

- Can similarities and differences between the mlearning preferences of students within different national groups be elicited?

Our expected deliverables are as follows:

- Understanding of the similarities and differences between the MLPs of students within different national cultural groups.

- Software design of an m-learning application to accommodate the MLPs of different learners within different national cultural groups.

The research methodology for this objective includes a questionnaire data collection method, which is distributed online and via possible collaborators in the different proposed countries. This questionnaire is developed based on our proposed model of MLPs. The goal is to obtain as large of a data sample as possible from university students of the different proposed countries. A further in-depth interview study methodology will then be conducted on students of a (possibly smaller) selection in the proposed countries. This is so that more detailed responses can be obtained from particular individuals or groups to answer more directly and thoroughly our research question; the interview studies will be conducted by a researcher onsite.

\section{CONCLUSION}

In this paper, we have proposed a model of MLPs consisting of five dimensions - location, level of distractions, time of day, level of motivation and available time. At the time of writing, no existing MLPs model has been identified. The use of learning preferences or MLPs in mlearning situations and/or applications are equally important, if not more, due to the non-stationary nature of mlearning. Therefore, the construction and validation of such a model will contribute significantly to the pedagogical aspects of m-learning We are currently conducting two research investigations -1 ) extension and validation of the model and 2) elicitation of similarities/differences of MLPs between learners of different national cultural groups.

\section{REFERENCES}

[1] Honey, P. (2001) Honey and Mumford Learning Styles Questionnaire. Available at: http://www.peterhoney.com/product/learning $\underline{\text { styles }}$

[2] Dunn, R. and Dunn, K (1978) Teaching students through their individual learning styles. A practical approach. Reston, VA: Prentice-Hall.

[3] Dunn, R. (1990) Rita Dunn answers questions on learning styles. Educational Leadership, pp. 15-19.

[4] Karagiannidis, C. and Sampson, D. (2004) Adaptation rules relating learning styles research and learning objects metadata. International Conference on Adaptive Hypermedia and Adaptive Webbased Systems.

[5] Graf, S., Yang, G. and Liu, T. (2009) Automatic, global and dynamic student modeling in a ubiquitous learning environment. International Journal of Knowledge Management \& E-learning, vol. 1, no. 1, pp. 18-35.

[6] Martin, E. and Carro, R. (2009) Supporting the development of mobile adaptive learning environments: a case study. IEEE Transactions on Learning Technologies, vol. 2, no. 1, pp. 23-36. doi:10.1109/TLT.2008.24

[7] Yau, J. and Joy, M. (2009) A mobile context-aware framework for managing learning schedules - data analysis from an interview study. International Journal of Mobile and Blended Learning, vol. 1, no. 4, pp. 29-55.

[8] Yau, J. and Joy, M. (2010) A context-aware personalized mlearning application based on m-learning preferences. International Conference on Wireless, Mobile and Ubiquitous Technologies in Education.

[9] Graf, S. and Kinshuk (2008) Adaptivity and personalization in ubiquitous learning systems. A. Holzinger (Ed.): USAB 2008, LNCS 5298, pp. 331-338. Springer-Verlag Berlin Heidelberg 2008.

[10] Dunn, R., Beaudry, J. and Klavas, A. (2002) Survey of research on learning styles. California Journal of Science Education - what we know about how people learn, vol. 2, issue 2, pp. 75-98.

[11] Chen, Y. S., Kao, T. C., Sheu, J. P. \& Chiang, C. Y. (2002) A mobile scaffolding-aid-based bird-watching learning system. International Workshop on Mobile Technologies in Education, pp. 1523.

[12] Chen, Y. S., Kao, T. C., Yu, G. J. \& Sheu, J. P. (2004) A mobile butterfly-watching learning system for supporting independent learn-ing, International Workshop on Mobile Technologies in Education, pp. 11-18.

[13] Chen, C. H., \& Chou, H. W. (2007) Location-aware technology in Chinese language learning. IADIS International Conference Mobile Learning, pp. 189-193.

[14] Chen, C. M., Li, Y. L. \& Chen, M. C. (2007a) Personalized context-aware ubiquitous learning system for supporting effectively English vocabulary learning. International Conference on Advanced Learning Technologies, pp. 628-630.

[15] Chen, C. M., Huang, T. C., Li, T. H., Huang, C. M. (2007b) Personalized e-learning system with self-regulated learning assisted mechanisms for promoting learning performance. International Conference on Advanced Learning Technologies, pp.637-638.

\section{AuTHORS}

J. Y-K. Yau is with the University of Warwick, Coventry, UK (e-mail: j.y-k.yau@warwick.ac.uk).

M. S. Joy is with the University of Warwick, Coventry, UK (e-mail: m.s.joy@warwick.ac.uk).

This article was modified from a presentation at The International Conference on Computer Aided Learning, ICL2010, held September 2010, at Hasselt University, Hasselt, Belgium.

Manuscript received, September 10, 2010. Published as resubmitted by the authors September 27, 2010. 